\title{
Hematuria Esencial de Origen Glomerular
}

\author{
Dr. Lugenio Rodríguez \$. ${ }^{1}$; Dr. Heimar Rosenberg G. ${ }^{2}$; Dr. Enzo Tassara P. ${ }^{3}$; \\ Dra. Katica Rartírez P. 3; Dr. Car]os Saieh A. ${ }^{1}$; Dr. Federico Puga C. ${ }^{1}$ \\ Idiopatic Recurrent Hematuria in Children
}

\begin{abstract}
Thirty two patients presenting with idiopatic recurrent hematuria in a ten years period (1972 through 1982) were submitted to renal biopsy and studied by optical, electronic and inmunefluorescense microscopy. The hystological findings were those of Berger's dizease in 7 (21.5\%) cases; Mininal change-glomerular disease was present in 21 patients $(66 \%$ and Thin Basal Membrane syndrome occurred in 4 (12.5\%) subjects. Generally speaking prognosis of idiopatic recursent hematuria is good in children and renal biopsy is rarely required unless further progress to chronic tenal failure, as described in some cases of Berger's disease, is suspected.

(Key words: Hematuria. Benign. Recurrent. Berger's disease. Thin basal membrane. Minimal change glomerular disease).
\end{abstract}

La hematuria en la infancia puede ocurrir en forma rislada o como parte de enfemedades especificas ${ }^{1,2}$. Constituye un signo alarmante para el pediatra y los padres, recurriéndose frecuentemente a procedimientos invasivos innecesarios para precisar su causa ${ }^{4}$.

Su origen puede estar en el parénquima renal o en la vía excretora denominándolas hematurias médicas o urológicas respectivamente ${ }^{8}$.

Se ha señalado que la morfología đel glóbulo rojo en el sedimento de orina, observado en el microscopio de fase contrastada, puede orientar hacia el origen de la hematuria ${ }^{\mathbf{3}, 5}$. Así, en hema. turias de origen glomerular se observa gran dismorfismo en los glóbulos rojos, en cambio si provienen de la vía excretora (cálices, pelvis, uréteres, etc.) la morfología del glóbulo rajo es similar a la sànguínea $y$ por ende con gran isomorfismo.

Las hematurias médicas pueden ser primarias, cuando se originan en el parénquima renal y su causa no es aparente, o secundarias a una enfermedad general con repercusión renal. Entre las hematurias primarias se encuentran las denominadas benignas, recurrentes o idiopáticas, nombres todos para definir episadios de hematuria macroscópica, alternados con períodos intercrí. ticos de hematuria microscópica o sin ella, ambos

1. Unidad de Nefrourología, Hospital Dr, Luis Calvo Mackenna.

2. Departamento Anatomía Patológica. Universidad Católica de Chile.

3. Becados de Pediatria, Hospital Dr. Luis Calvo Mackensa. de duración variable y de origen desconocido. Se presenta en ambos sexos, desde el año de vida a la edad adulta y generalmente es precedida por síntomas respiratorios altos, ejercicios intensos o enfriamientos bruscos. La función renal, los estudios radiológicos y morfológicos habituales son normales ${ }^{6,7}$.

Los objetivos de este estudio son: presentar una serie de 32 niños con hematuria de origen glomerular y que fueron estudiados con métodos clínicos, funcionales, rađiológicos y morfológicos. Analizar morfológicamente, mediante el microscopio electrónico, la presencia de sub-grupos histopatológicamente diferentes, observando su presentación clínica, evolución y pronóstico. Precisar la indicación de biopsia renal en esta afección a la luz de los resultados obtenidos.

\section{MATERIAL Y METODOS}

Entre los años 1972 y 1982, en la Unidad de Nefrología del Hospital Luis Calvo Mackenna, fueron realizadas 232 biopsias renales por hematurias médicas, excluyendo nefritis aguda, púrpura de SchöenkinHenoch, síndrome hemolítico urémico, lupus eritematoso sistémico y síndrome de Alport. En todos los pacientes se descartaron malformaciones renales y de las vías uninarias mediante pielografias descendentes $y$ uretrocistografias retrógradas.

\section{RESULTADOS}

Se analizaron 76 biopsias renales mediante microscopia de luz, electrónica y de inmunofluo. rescencia, de las cuales 32 reunieron las caracte- 
risticas clinicas de hematuria benigna recurrente $y$ tuvieron un seguimiento adecuado. De los 32 pacientes estudiados, $13(41 \%)$ eran mujeres. No se encontraron diferencias significativas en la distribución por sexo. El mayor número de pacientes se acumuló en el grupo etáreo de 6 a 7 años (Figura 1).

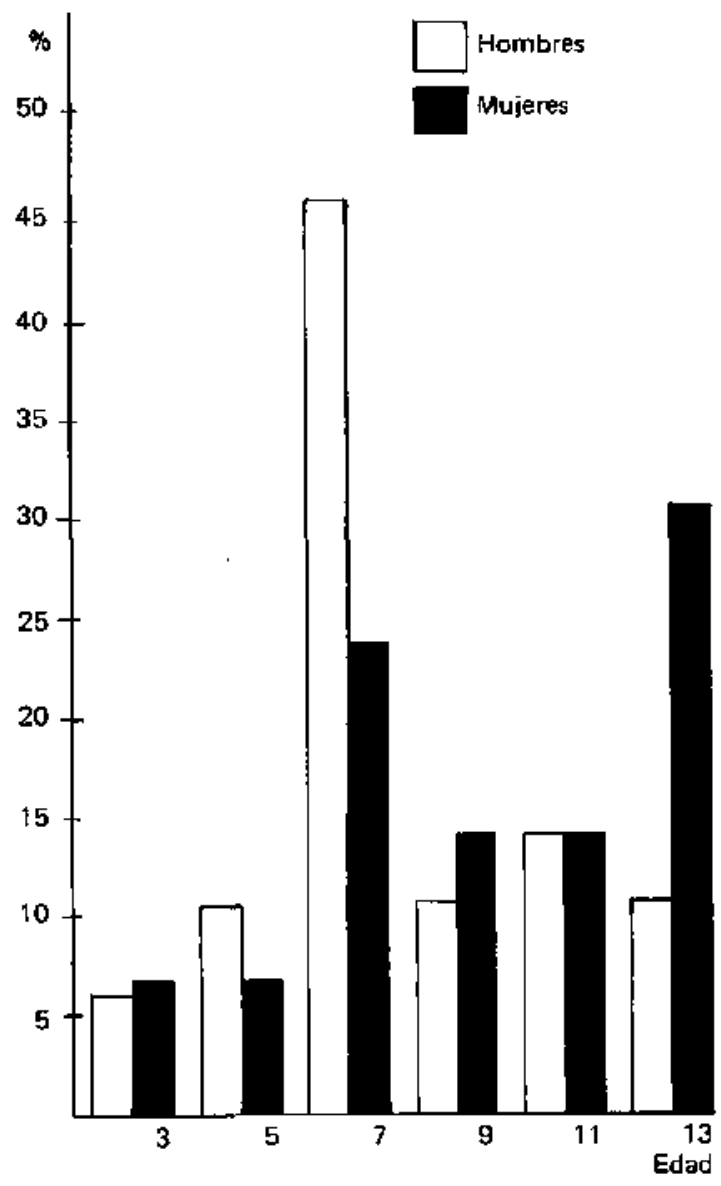

Fisura 1. Distribución porcentual por sexos y edad de 32 niños con hematuria esencial.

De acuerdo a la modalidad clínica de presentación, 17 pacientes $(53,1 \%)$, presentaron episodios de hematuria micro o macroscópica con una frecuencia mayor de 3 al año. La duración de la hematuria no excedió los 7 dias en 30 pacientes $y$ en dos de ellos se hizo permanente.

La distribución según el tiempo de evolución antes de la biopsia renal y de acuerdo con los hallazgos morfológicos se describen en la Tabla 1.

Los hallazgos hechos mediante microscopía óptica (MO), electrónica (ME) y de inmunofluorescencia (MI) se pueden clasificar en tres grandes grupos:

Enfermedad de Berger (Grupo 1): Siete pacientes $(21,5 \%)$ tenian glomerulonefritis con
Tabla 1 .

Hematuria esencial de origen glomerujar. Tiempo de evolución antes de h biopsia renal según diagnóstico moffológico en 32 pacientes.

\begin{tabular}{lccc}
\hline Meses & Berger & A.M.G. & M.B.D. \\
\hline $0-10$ & 4 & 11 & 2 \\
$11-20$ & & 4 & 1 \\
$21-30$ & & 4 & \\
$31-40$ & 2 & 1 & 1 \\
$41-50$ & 1 & & \\
$51-60$ & & 1 & \\
$61-70$ & 7 & 21 & 4
\end{tabular}

depósitos mesangiales de IgA-lgG difusos y generalizados (Ml) y depósitos densos mesangiales en la microscopía electrónica.

Alteraciones glomerulares mínimas (Grupo 2): Se encontraron en 21 niños de la serie $(66 \%)$. Hemos sub-dividido estos hallazgos en 3 sub-grupos:

Lesiones inespecificas (Sub-grupo 2a): En estos casos la observación mediante microscopio óptico es normal, con alteraciones minimas glomerulares. La inmunofluorescencia es negativa $y$ la microscopía electrónica muestra leves alteraciones, consistentes en aumento de la matriz mesangial, con o sin alteración de los pliegues de la membrana basal paramesangial. Esta forma se encontró en 15 pacientes $(71,5 \%)$.

Membrana basal irregular (Sub-grupo 2b): De sinilares caracteristicas al grupo anterior mediante c] estudio de la microscopía de luz e inmuno. fluorescencia. La microscopía electrónica demuestra irregularidad discreta de la membrana basal a paramesangial y ocasionalmente leve retracción del mesangio. Estos hallazgos ocurrieron en 2 niños $(9,5 \%)$.

Depósitos del factor 3 del complemento hemolitico (Sub-grupo 2c): Este fenómeno se produce en este sub-grupo sólo en las arteriolas, especialmente en el polo vascular glomerular, con compromiso de la intima y la túnica media. La inmunofluorescencia es negativa en los glomérulos. La microscopía óptica no muestra alteraciones o éstas son mínimas y en la microscopia electrónica se observan leves alteraciones mesangiales con membrana basal irregular, similar en el sub-grupo anterior. Cuatro pacientes $(\mathbf{1 9 \%})$ pertenecen a este sub-grupo.

Membrana basal delgada (Grupo 3): Definida por la detección, mediante microscopia electrónica, de asas capilares cuya membrana basal mide menos de 2.000 Angström de espesor en especímenes renales cuyo estudio con microscopia de luz e inmunofluorescencia es normal. Se pesquisaron 4 pacientes con estas caracteristicas $(12,5 \%)$. 
El tiempo minimo de seguimiento fue de 4 meses y el máximo 120. Su duración es decrita para cada caso en la Tabla 2. En ninguno de estos pacientes se detectó proteinuria, hipertensión arterial ni deterioro de la función renal.

\section{COMENTARIO}

Nuestro criterio para efectuar biopsia renal en esta afección, ha cambiado con el transcurso del tiempo. En los últimos 5 años sólo se estudiaron morfológicamente niños que tuviesen más de dos años de hematuria microscópica persistente 0 recurrente, hematuria macroscópica permanente por más de un mes, asociación con proteinuria significativa durante la evolución, hipertensión arterial permanente o compromiso de la función renal.

Este estuđio no mostró diferencias significativas por sexo en la frecuencia del trastorno. El mayor número de pacientes se distribuye entre las edades de 5 y 7 años $(37,5 \%)$ repartiéndose en forma homogénea en los años posteriores hasta los 13 años.

No se observaron diferencias significativas en cuanto a la expresión clínica de la hematuria: sin embargo los pacientes con enfermedad de Berger en su mayoría tuvieron 3 o más episodios de hematuria macroscópica por afio. El sindrome de membrana basal delgada se puede presentar en diferentes formas clínicas, con episodios de he. maturia macroscópica o microscópica frecuentes aisladas y ocasionalmente permanentes. Las alteraciones mínimas tampoco se manifiestan de una manera específica: los episodios pueden ser frecuentes o ên largos intervalos y aún como hema. turia permanente.

Los resultados de esta serie en general concuerdan con otros en lo referente al tipo de lesión morfológica y la forma de presentación clinica ${ }^{9.10}$.

En general la hematuria en la infancia, cuando es aislada, sin dolor ni hipertensión arterial ni alteración de la función renal, en la gran mayoría de los casos es de origen glomerular y no es posible identificar su causa. La evolución clínica de estos pacientes no permite distinguir los di- ferentes grupos que pueden ser identificados en el estudio morfológico de la biopsia renal.

La histología renal de niños con hematuria debe estudiarse mediante las tres técnicas clásicas pues la microscopia óptica por si sola no es capaz de distinguir ningún sub-grupo, la inmunofluorescencia es capaz de diferenciar la enfermedad de Berger y el grupo con arteriolas $\mathrm{C} 3$ positivo $y$ la microscopía electrónica permite distinguir el sindrome de la membrana basal delgada y la glomerulopatia con depósito mesangial. Asumismo el estudio morfológico permite descartar otras enfermedades glomerulates como la nefritis aguda; el síndrome de Alport, el púrpura de Schöenlein-Henoch, etc.

La hematuria esencial en la infancia en general, tiene buen pronóstico y las diferencias mor. fológicas que se encuentran son sutiles, por tanto debe seleccionarse con la mayor acuciosidad cada paciente antes de efectuar una biopsia renal.

Tanto la enfermedad de Berger como en sindrome de membrana basal delgada deben con. trolarse por un largo tiempo pues algunos de estos pacientes han evolucionado hacia la insuficiencia renal crónica ${ }^{9}$. Asimismo se postula en la actualidad que el síndrome de membrana basal delgada es un precursor del síndrome de Alport, no acompañado de alteraciones auditivas ni oculares 10 .

\section{RESUMEN}

Se describen 32 niños con hematuria recurrente idiopática, de quienes se tomaron biopsias renales que fueron estudiadas morfologicamente mediante microscopio óptico, electrónico e inmunofluorescencia en un hospital de Santiago entre los aftos 1972 a 1982 . En $21,5 \%$ de los casos se encontró enfermedad de Berger, en $66 \%$ alteraciones mínimas glomerulares y en $12,5 \%$ membrana basal delgada glomerular.

Se propone una clasificación en sub-grupos para las alteraciones mínimas glomerulares.

Se concluye que esta es en general una afeccion benigna en la infancia, que habitualmente no requiere de biopsia renal, con excepción de la

Tabla 2.

Hematuria Esencial de Origen Glomenular. Tiempo de Seguimiento de 32 Pacientes según Diagnóstico Morfológico.

\begin{tabular}{lcccccc}
\hline Meses & $1-24$ & $25-48$ & $49-72$ & $73-96$ & $97-120$ & Total \\
\hline Berger & 3 & 1 & 1 & 1 & 1 & 7 \\
\hline A.M.G. & 10 & 5 & 3 & 2 & 1 & 21 \\
\hline M.B.D. & & 1 & 1 & 1 & 1 & 4 \\
\hline \hline
\end{tabular}


enfermedad de Berger y la membrana basal de1gada en los que se ha descrito excepcionalmente progresión a insuficiencia renal.

\section{REFERENCIAS}

1. Wyatt R., Roberts J.W., Holkand N.: Hematuria in Childhood. Significance and management. J. Urol. 117: 866,1977 .

2. Lieberman E.: Workup of the child with hematuria. Clinical pediatric Nephrology, J.B. Lippincott Co. Philadelphia, 1976. Pág. 12-26.

3. Fairley K.F., Doyglas B.: Hematuria: A simple Method for identify in glomerular bleeding. Kidney Int. 21: 105, 1982 .

4. West C.: Asymptomatic Hematuria and proteinuria in children: Canses and appropiate diagnostic studies. J. Pediatr. 89: 173, 1976.

5. Birch D.F., Fairley K.F.: Hematuria: Glomerular or non-glomerular? Lancet 2: 845, 1979.

6. Chan J.C.: Hematuria and Proteinuria in pediatric patients: Diagnostic approach. Urology 11: 205, 1978.

7. Yemakasri M., Rapola J., Koskimies o. et cols.. Microscopic hematuria in school children; epidemiology and clinic pathologic evaluation. I. Pediatr. 95: 676,1979 .

8. Northway J.D.: Hematuria in Children. J. Pediatr. 78: 381,1971 .

9. Grinswold W'W.: Hematuria in Children. Dialogues in Pediatric Urology 8: 4, 1985.

10. Greifer 1: Clinical pathological aspects of hematuria in children and adolescents. Comunicación personal. I Congreso Latinoamericano de Nefrologia Pediátrica. Vîna del Mar, Octubre 1984. 